\title{
In situ mechanical and electrical characterization of composite-integrated thin film-supercapacitors
}

\author{
Jan Petersen ${ }^{1, *}$, Sebastian Geier ${ }^{1}$, Peter Wierach ${ }^{1}$ and Martin Wiedemann ${ }^{1}$ \\ ${ }^{1}$ DLR German Aerospace Center, Institute of Composite Structures and Adaptive Systems, 38108 \\ Braunschweig, Germany
}

\begin{abstract}
Future transport systems will be powered more and more electrically. Generally the energy is stored in batteries. To reduce system weight and volume, multifunctional materials could be the answer. Therefore materials with the capability to store electric energy and to bear mechanical loads, need to be investigated to understand the effect of mechanical load on such structural integrated energy storage devices. In this work a thin film-supercapacitor is build up and integrated within a composite structure. The capacitor is developed to withstand the manufacturing process of a glass-fibre reinforced polymer and to carry mechanical loads, while simultaneously storing electrical energy. By using a supercapacitor housing, which is compatible to epoxy resin, a strong bonding is achieved, leading to a mechanical robust multifunctional composite. An electrolyte with large temperature window, low vapour pressure and the compatibility to a carbon based electrodes is chosen, to meet the requirement regarding the manufacturing process of the supercapacitor itself and the fibre reinforced composite. The composites with integrated thin film-supercapacitor as well as a set of reference samples are mechanically characterised in tensile and four-point bending test. In situ measurements are performed to investigate the influence of mechanical load on the electrical performance.
\end{abstract}

\section{Introduction}

Nowadays an increasing amount of products are equipped with electric systems, leading to a higher system weight and volume, e.g. assistance systems and consumer electronics in automotive and aviation [1,2]. For the purpose of buffering electrical energy, providing electrical systems in standby, or allowing electrical peak power, devices like batteries and/or (super)capacitors are being used more often. Those energy storage devices normally persist of approximately $30 \mathrm{wt}-\%$ of housing material, which is parasitic for the overall system. An approach to reduce system mass and volume is to substitute parts of the electric storage by structural, load bearing, elements. Through integrating energy storage devices into structures, huge benefits can be gained such as decentralising electronic systems, reduction of assembly time or reduction of wiring and cable weight. Parts of the structure can undertake functions of the energy storage e.g. acting as housing components while energy storage materials also bear loads.

\footnotetext{
* Corresponding author: Jan.Petersen@dlr.de
} 
In this study thin film supercapacitors are integrated in glass fibre reinforced composites. A supercapacitor is an electrochemical storage device which is capable of providing and storing electric energy ten times faster than batteries [3]. This can be achieved by reversible charge separation in Helmholtz double layers making the supercapacitors very durable, which is an important aspect for a long-life maintenance-free multifunctional material [4].

\section{Experimental}

\subsection{Sample preparation and cell assembly}

The thin film-supercapacitor, integrated in this work, is produced by coating carbon based electrodes (mixture of carbon black and carbon nanotubes) onto thin copper current collectors. Two electrodes are assembled to one supercapacitor. To avoid shortening of the electrodes, a polycarbonate membrane is used as separator, soaked in an ionic liquid (EMIBF4). To enclose the supercapacitor, a polyimide film is used. The housing is sealed under vacuum and closed with epoxy resin. A schematic drawing of the final supercapacitor is illustrated in figure 1 . In case of tensile and four-point bending, the sample dimensions are, length $240 \mathrm{~mm}$, width $15 \mathrm{~mm}$, thickness $2 \mathrm{~mm}$ and length $50 \mathrm{~mm}$, width $5 \mathrm{~mm}$, thickness $2 \mathrm{~mm}$, respectively. The Integration is done, inserting the capsuled supercapacitors in the neutral axis of a GFRP prepreg stack, consisting of 16 layers. The thickness of a supercapacitor is equal to one layer of prepreg. For tensile and four-point bending, reference samples with a total of 17 layers are manufactured. Curing is done in an autoclave process at $120^{\circ} \mathrm{C}$ for $1 \mathrm{~h}$ at a pressure of 6bar. The final geometry according to the standard $[5,6]$ is reached by cutting the samples.

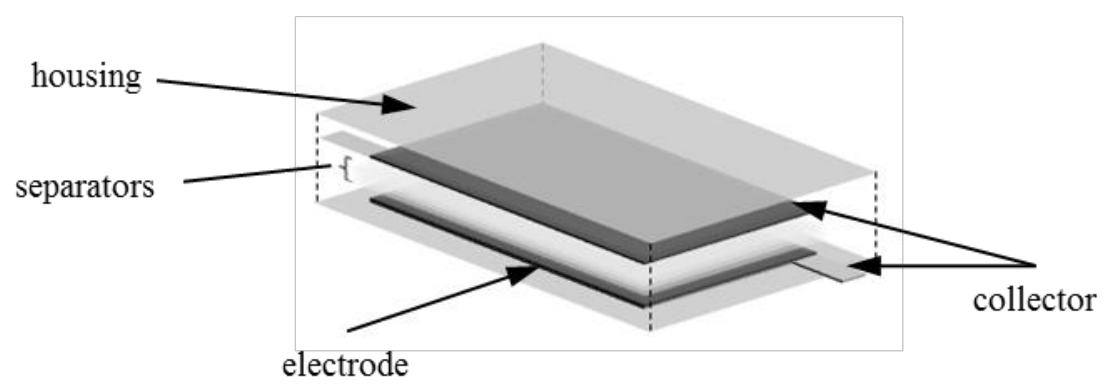

Fig. 1. Design of the pouch cell

\subsection{Electrochemical tests}

The integrated thin film-supercapacitor is characterised with an electrochemical workstation (Zennium-pro, Zahner-Elektrik GmbH \& Co. KG, Germany). Cyclic voltammetry is performed to obtain the electric performance of the energy storage during the mechanical tests. For each specimen, three cycles of $\mathrm{CV}$ are performed. During the electrical characterisation the stress is held at constant level. 


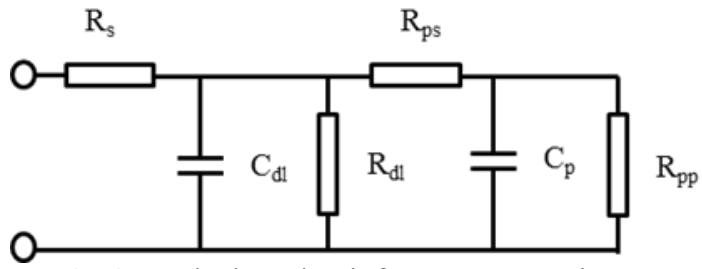

Fig. 2. Equivalent circuit for a supercapacitor.

In order to understand the results of the cyclic voltammetry with respect to mechanical load a simplified equivalent circuit is build up (Fig.2, $[4,7,8]$ ) and modelled with the software LTspice (LTspice, Analog Devices, USA). The model is fitted to reach a congruent result compared to the result of the cyclic voltammetry recorded during the mechanical experiments.

$\mathrm{C}_{\mathrm{dl}}$ represents the capacity responsible for the fast charge and discharge behaviour while $\mathrm{C}_{\mathrm{p}}$ stands for slow capacities e.g. small pores. $\mathrm{R}_{\mathrm{ps}}$ is a serial resistance arising from diffusion process while $R_{s}$ describes resistances from diffusion and electrodes resistance. $\mathrm{R}_{\mathrm{pp}}$ and $\mathrm{R}_{\mathrm{dl}}$ are responsible for self-discharge and short circuit current. With a decrease in $\mathrm{R}_{\mathrm{dl}}$ and/or $\mathrm{R}_{\mathrm{pp}}$ the $\mathrm{CV}$ curve will tend to be skewed (Fig. 3b) leading to a lower $\mathrm{C}_{\mathrm{dis}} / \mathrm{C}_{\mathrm{ch}}$ ratio as more of the $\mathrm{CV}$ curve is located in the area of positive current. This is illustrated in Fig. 3b).
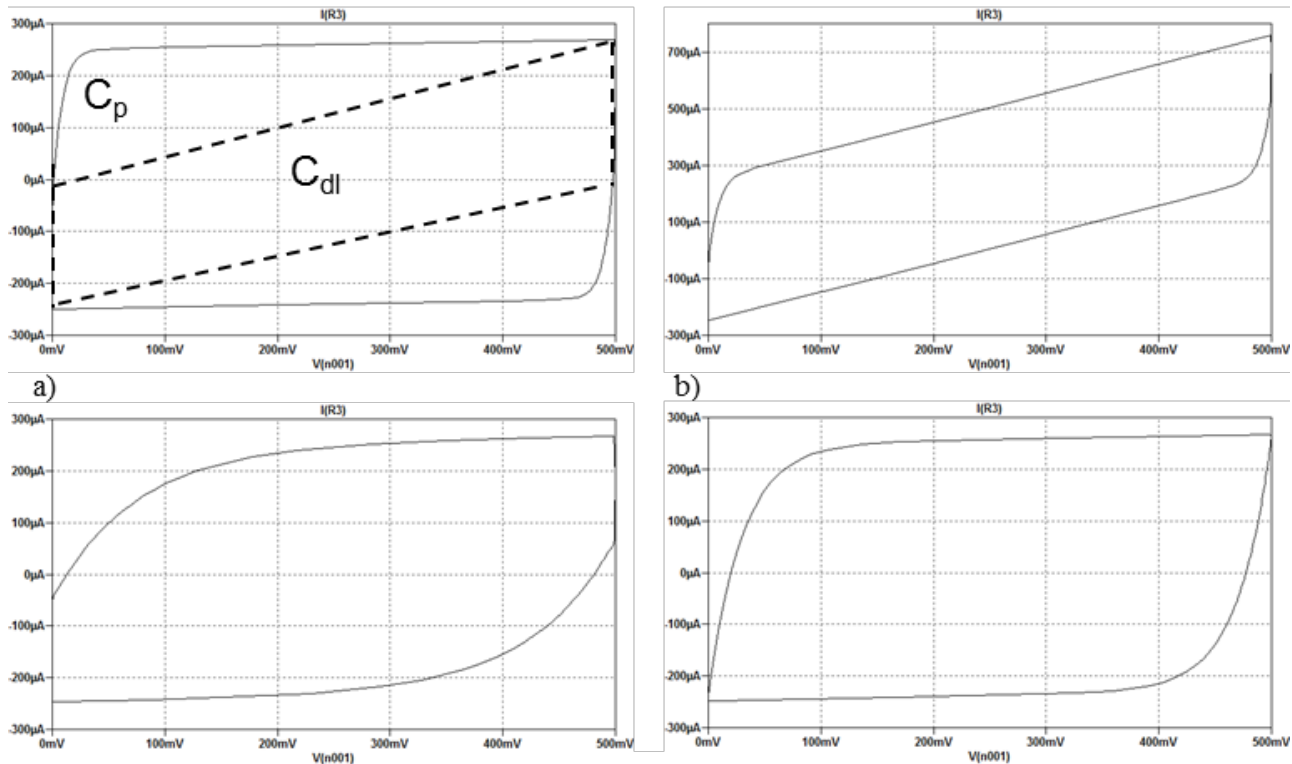

c)

d)

Fig. 3. Results of the simulation model to describe the $\mathrm{CV}$ in dependence of the equivalent circuit a) baseline with $C_{d l}$ (white zone) and $C_{p}$ (surrounding area) b) change of $R_{d l} c$ ) change of $R_{p s} d$ ) change of $\mathrm{R}_{\mathrm{s}}$

By increasing $R_{s}$, the vertical lines (at $0 \mathrm{~V}$ and $0,5 \mathrm{~V}$, Fig. 3) begin to rotate clockwise (Fig. 3d)) and change their shape from a straight line to a more round curve. $R_{p s}$ is responsible for the radius of the curves located in the upper left and on the bottom right (Fig. 3c)). By increasing $\mathrm{R}_{\mathrm{ps}}$ the radius becomes larger too. $\mathrm{C}_{\mathrm{dl}}$ and $\mathrm{C}_{\mathrm{p}}$ is defining the area 
(Fig. 3a)) limited by the curves. The field within the white zone is due to $\mathrm{C}_{\mathrm{dl}}$, the remaining area due to $\mathrm{C}_{\mathrm{p}}$.

\subsection{Mechanical tests}

The mechanical investigation is done using two testing machines. For tensile tests a Zwick 1476 is used, while four point-bending is done, using a Zwick 1484.

Tensile measurements are carried out with a 100kN load cell (BZ2-MM14760-ZW02, Zwick GmbH \& Co KG, Germany), strain gauges (DMS, XY-F/DMS, Höttinger Baldwin Messtechnik GmbH, Germany) and an extensometer (MTS 776, model 632.85, MTS Systems Corporation, USA). All four point-bending tests are done using a $5 \mathrm{kN}$ load cell (type BZ2-MM14760.ZW02, Zwick GmbH \& Co KG, Germany) and a linear position sensor (WA 50, type K-WA-U-050-W-32K-K4-F1-2-8-10, Höttinger Baldwin Messtechnik $\mathrm{GmbH}$, Germany).

To enable the in situ electrochemical measurement, steps of constant load are implemented. During those steps the measurement is done. The steps for tensile testing are located every $10.000 \mathrm{~N}$ while load steps for four point-bending are located every $250 \mathrm{~N}$. For the reason of comparison one sample of each (tensile and four-point bending) is tested without constant load steps until failure, like all the reference ones. Specimens conditioning and mechanical testing is performed at $23^{\circ} \mathrm{C}$ and $50 \%$ rh according to the standard $[5,6,9]$. Every test series consists of four samples.

\section{Results and discussion}

\subsection{Equivalent circuit model}

To understand the mechanical impact on the electrical behaviour, a simple equivalent circuit is build up [4], which defines fast and slow capacities, characteristic for carbon based electrodes. The two parallel resistances $\left(R_{d l}, R_{p p}\right)$ are used to describe the selfdischarge process of the supercapacitor, while the serial resistance $R_{s}$ is correlating for losses, regarding the current collector, collector/electrode interface and the losses from ion diffusion. $R_{s}$ is linked to the fast capacity, $R_{p s}$ to the slower one.

\subsection{Electrochemical investigation}

Electrochemical investigation is done using cyclic voltammetry. The cyclic voltammetry measurements are set to a scanrate of $50 \mathrm{mV} / \mathrm{s}$ with a potential window of $0 \mathrm{~V}$ to $+0,5 \mathrm{~V}$. The results of the $\mathrm{CV}$ measurement are used to analyse the impact of mechanical load on the electric behaviour.

Two cyclic voltammetry are shown in figure 4. Part a) visualises the response of an integrated thin film-supercapacitor in a tensile specimen, part b) is showing the influence of bending load. Both measurements are done at zero load and at constant load. In case of tensile and bending it is $50.000 \mathrm{~N}$ (load step 5) and $1250 \mathrm{~N}$ (load step 5), respectively.

Through the positive lateral strain of the glass fibre composite a compression of the integrated thin film-capacitor is arising as a result of the transversal contraction [10]. This leads to a reduction in the electrode/electrode distance, influencing the serial resistance $R_{s}$ of the equivalent circuit. With increasing the tensile force, a more ideal cyclic voltammetry is to be expected, but the results are showing a less ideal behaviour. The curve tends to be more round, like shown in figure $3 \mathrm{~d}$ ). This is indicating a gain in serial resistance $\mathrm{R}_{\mathrm{ps}}$ and therefore a reduced access to small diameter pores. Through the tensile load on the 
specimen a transversal contraction of the supercapacitor, more specific the electrode occurs. The connection between ion diffusion into electrodes and mechanical load is described by Geier et al [10]. They investigated paper-like specimens based on carbon materials, similar to the supercapacitors electrode described in this work. They assumed that the access of ions in a porous structure can be influenced by preloading [11].

In four-point bending tests, no reduced access of ions related to mechanical load could be observed. This can be explained by the different type of load on the specimen, respectively the supercapacitor and its electrode. As the supercapacitor is located in the neutral axis and its thickness is low compared to the overall thickness of the laminate, only compression and shear load is occurring and therefore no transversal contraction, which could lead to a reduced access of ions to the pores.

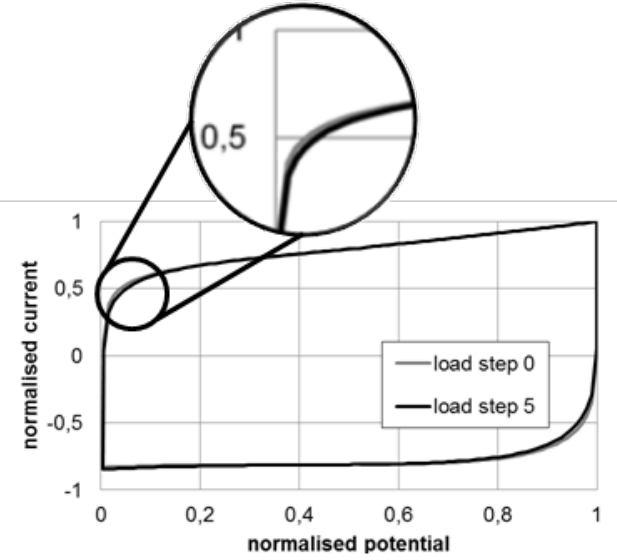

a)

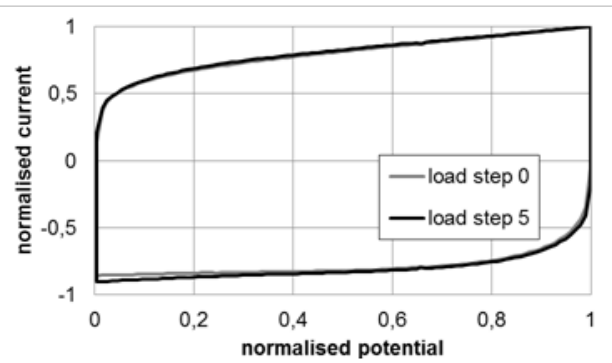

b)

Fig. 4. a) Normalized CV of a integrated thin film supercapacitor (TEN 04) in tensile test with a scanrate of $50 \mathrm{mV} / \mathrm{s}$. b) CV of a ITFC (4PB 02) in 4PB test with a scanrate of $50 \mathrm{mV} / \mathrm{s}$.

Nevertheless a load dependent influence of bending load on the electric performance is present (figure $4 b$ )). Like described earlier (figure 3), the CV is indicating a reduction in the serial resistance. With increasing bending load, the supercapacitor is being compacted and therefore the distance between the electrodes is decreasing. This reaction leads to a higher force $(\mathrm{F})$ on the ions, because of the proportion between the electric field (E) and the electrodes distance $(\mathrm{d})(\mathrm{E}=\mathrm{U} / \mathrm{d}=\mathrm{F} / \mathrm{q}$ [12]). The mobility of the ions is enhanced and therefore the serial resistance is decreased.

Cyclic voltammetry is done during the mechanical test. Figure 6 is showing two CV curves before and after the mechanical failure of the overall system. The electric performance of the supercapacitor before the failure is illustrated in grey, after the failure the curve is shown in black. Two different types of electric response due to failure of the system could be observed, shown in figure 6 a) and b). One failure recorded is an abrupt loss of current. Different mechanism of failure could be responsible. First a damage of the current collector, second a loss of the interface between electrode and current collector and third a rapid change in distance between the electrodes. The third option is unlikely, as the supercapacitor is fixed in a composite structure. In case of interface damage, an increase in serial resistance will appear, but no loss of current as the electrode is still in contact with the collector. The first option is most likely. Through the mechanical tension in the composite, a transversal crack might have appeared, leading to a damage in the current collector and therefore a loss of current. 
The other mechanism of failure is a sudden increase in current, with a joining normal current response. The increase appears at $0,4 \mathrm{~V}(0,8$ normalised potential) simultaneously with the mechanical failure. A mechanical shockwave as a result of the failure of the specimen might have occurred, which could cause a decrease in the electrodes distance. Like described earlier, this leads to a reduction in serial resistance, resulting in an increase in current. Another solution for the sudden current peak, is a decrease of parallel resistance also triggered by a mechanical shockwave, leading to a short circuit for a small period of time.

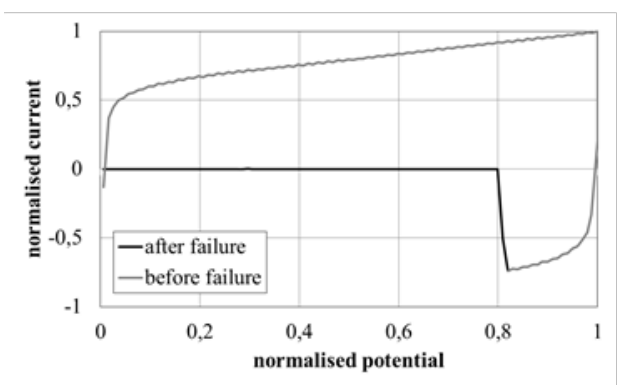

a)

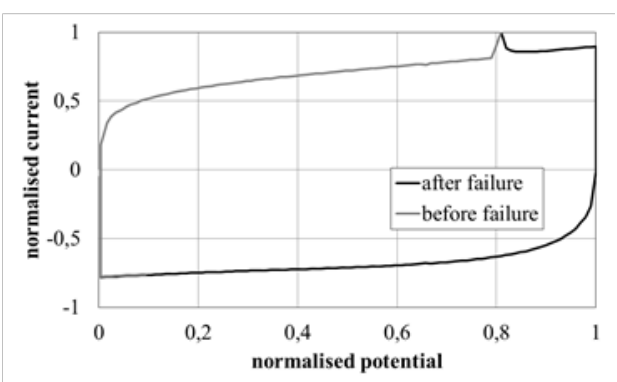

b)

Fig. 5. $\mathrm{CV}$ of an ITFC in $4 \mathrm{~PB}$ test, at the moment of the failure a) specimen $4 \mathrm{~PB} 04$ at a scanrate of $500 \mathrm{mV} / \mathrm{s}$. b) specimen $4 \mathrm{~PB} 02$ at a scanrate of $50 \mathrm{mV} / \mathrm{s}$.

\subsection{Mechanical investigation}

The test procedure for samples with integrated energy storage is a modified version of the standard with additional steps of constant load. Tensile test are being done to examine the influence of the supercapacitor on the tensile strength and elastic modulus. The change in bending modulus as well as the bending strength is investigated in four-point bending. To transfer all shear stress through the supercapacitor, its dimension is the same as the outer dimension of the specimen. The impact of the mechanical stress on the electrical performance is investigated in situ by cyclic voltammetry (chapter 3.2). For each specimen, tensile and four-point bending, one sample is being tested without load steps for the reason of comparison.

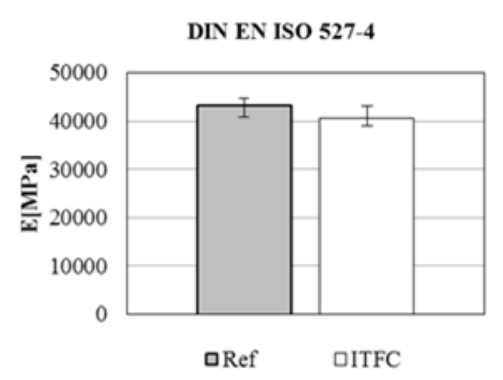

a)

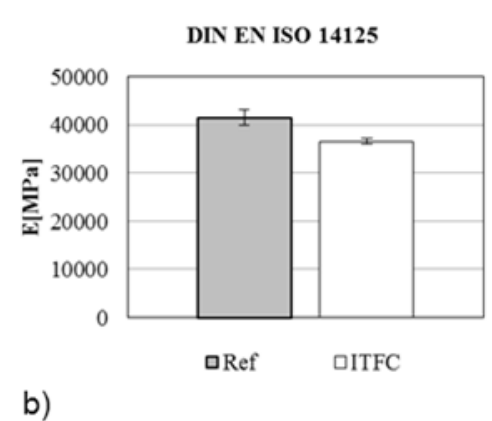

b)

Fig. 6. a) Tensile modulus (calculated at $0,05 \%-0,25 \%$ strain) of reference samples and ITFC samples. b) Flexural modulus (calculated at 0,2\% - 1\% strain). Error bar indicates the greatest and smallest value. An adjustment of the norm for Young's modulus calculation in case of 4PB is done. 
Figure 6 shows the results of the tensile and bending tests. Grey represents the average modulus of elasticity for the reference samples, white the modulus for the samples with integrated supercapacitor. The error bars are indicating the greatest and smallest value. Results of tensile test are shown in figure $6 \mathrm{a}$ ), while figure $6 \mathrm{~b}$ ) is presenting the results of the four-point bending tests.

In tensile, the modulus of elasticity is calculated according to the standard, through the secant method between $0,05 \%$ and $0,25 \%$ strain. In case of four-point bending, the area of secant method was adjusted to $0,2 \%-1 \%$ strain, to avoid settlement effects in the calculation.

For the reference samples in tensile, there is a modulus of elasticity of approximately 43.000MPa and a tensile strength of $1.100 \mathrm{MPa}$. Through the integration of a supercapacitor layer, the modulus of elasticity reduces by $6 \%$ while the tensile strength is reduced by $8 \%$. A more dominant behaviour exists in four-point bending. The average bending modulus is $41.000 \mathrm{MPa}$ with a bending strength of $1.050 \mathrm{MPa}$. By integrating a supercapacitor in the laminate, its bending modulus reduces by $12 \%$ while the bending strength is reduced by $18 \%$. Like shown in figure 6 scattering has not changed through the integration. A stronger reduction of the mechanical properties in four-point bending was to be expected, as all shear stress is transferred by the supercapacitor, compared to a shared load in tensile testing.

\section{Conclusions}

A manufacturing process to integrate thin film-supercapacitors in a composite structure is described in this paper. The supercapacitor is designed to be compatible to epoxy resin, creating a bond to the surrounding matrix. Additionally the thin film-supercapacitor is able to withstand the boundary conditions (temperature, vacuum) in an autoclave process, which are needed to cure the composite structure. A set of four tensile specimens and four bending specimens is manufactured for mechanical testing as well as electrical characterisation. For the reason of comparison a set of reference samples is manufactured without integrated supercapacitors. The electrical performance of the composite integrated supercapacitor is characterised during mechanical tests to investigate the influence of mechanical load onto the electrical system. To understand the electric response of the supercapacitor, the results of the measurement are compared to an equivalent circuit, modelled by software. A connection between tensile load and the access for ions in the electrode is being found. This leads to a load dependent ionic mobility. An improvement of ionic mobility through bending load was observed and discussed. Those two mechanisms of action can be separated in cyclic voltammetry and could be used in structural health monitoring. In situ measurements of the electric response due to mechanical load were done during the mechanical failure of the specimen. Furthermore the change in modulus of elasticity and bending through the integration of a supercapacitor is analysed. The modulus in tensilemode reduces by $6 \%$, while the modulus of bending is reduced by $12 \%$ showing promising load transmission.

Through the adaptable cell design presented in this work, the opportunity arises to integrate different components of supercapacitors, regarding energy and power density. Even the integration of batteries is possible, expanding the field of research. 


\section{References}

1. M. Sterner, I. Stadler, Energiespeicher - Bedarf, Technologien, Integration (Springer Vieweg, 2014)

2. A. Karle, Elektromobilität Grundlagen und Praxis (Carl Hanser Verlag München, 2017)

3. T. Christen, M.W. Carlen, Journal of Power Sources, 91, 210-216, (2000)

4. B. E. Conway, Electrochemical Supercapacitors - Scientific Fundamentals and Technological Applications (Kluwer Academic, 1999)

5. DIN EN ISO 14125, Faserverstärkte Kunststoffe - Bestimmung der Biegeeigenschaften, (Beuth Verlag GmbH, 1998)

6. DIN EN ISO 527-4, Kunststoffe - Bestimmung der Zugeigenschaften - Teil4: Prüfbedingungen für isotrop und anisotrop faserverstärkte Kunststoffverbundwerkstoffe, (Beuth Verlag GmbH, 1997)

7. S. Fletcher, V. Black, I. Kirkpatrick, J Solid State Electrochem, 18, 1377-1387, (2014)

8. C. Lämmel, M. Schneider, M. Weiser, A. Michaelis, Mat.-wiss. U. Werkstofftech, 44, 641- 649, (2013)

9. EN 62391-1, Elektrische Doppelschichtfestkondensatoren zur Verwendung in Geräten der Elektronik - Teil 1: Fachgrundnorm, (Electrosuisse, 2006)

10. H. Schürmann, Konstruieren mit Faser-Kunststoff-Verbunden (Springer, Berlin, 2007)

11. S. Geier, T. Mahrholz, P. Wierach, M. Sinapius, Smart Structures and Materials Experimental Investigations of Actuators Based on Carbon Nanotube Architectures, (Springer, 2016)

12. W. Demtröder, Experimentalphysik 2 - Elektrizität und Optik (Springer, 2009) 